
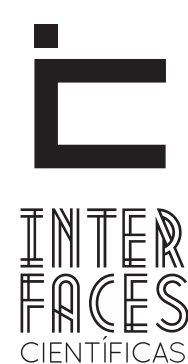

SA ÚDE E AMBIENTE

\title{
ASSISTÊNCIA DE ENFERMAGEM NAS COMPLICAÇ̃̃ES RESPIRATÓRIAS DA DISTROFIA MUSCULAR DE DUCHENNE
}

\author{
Mayanna machado freitas ${ }^{1}$ \\ Edmara oliveira mota ${ }^{1}$ \\ Gleyce kelle azevedo da $\mathrm{cruz}^{1}$ \\ Fernanda santos silva ${ }^{1}$ \\ Gleyce kelly santos cruz
}

\author{
Juliana gonçalves carvalho ${ }^{1}$ \\ Keyciane bispo de santa rita \\ Layane santos de menezes ${ }^{1}$ \\ Maricélia socorro jesus ${ }^{2}$ \\ Ana maria laurindo da silva ${ }^{3}$
}

\section{RESUMO}

A distrofia muscular de Duchenne corresponde a um grupo de doenças de origem genética progressiva que se caracterizam por alterações estruturais ou funcionais dos músculos. 0 tratamento de Enfermagem é manter a função dos níveis respiratórios e estimular a qualidade de vida. Nesse contexto, o objetivo do presente artigo foi verificar a atuação da enfermagem nas complicações respiratórias da DMD. Este trabalho consistiu em uma revisão bibliográfica, cuja estratégia de busca incluiu consulta à base de dados eletrônicas Scielo, Science Direct, Bireme e Pedro. A ausência de estudos envolvendo a prática de Enfermagem na Distrofia Muscular de Duchenne, a mais comum e mais grave dentre as doenças neuromusculares hereditárias conhecidas, tem levado a não tomada de atitudes coerentes por parte dos poderes públicos e privados, das sociedades médicas e científicas. Há a necessidade de se conhecer a atuação da Enfermagem nas complicações respiratórias da DMD, a fim de que este conhecimento possa contribuir para possíveis ações mais precisas e eficazes por parte de agentes públicos e privados que estão direta ou indiretamente envolvidos na prestação de serviços de saúde, bem como promover a formação de conhecimento crítico e humanístico que possam favorecer que estudantes e profissionais tenham informações verdadeiras e relevantes.

\section{PALAVRAS-CHAVE}

Distrofia Muscular de Duchenne. Enfermagem. Complicações Respiratórias. 


\section{ABSTRACT}

Duchenne muscular dystrophy is a group of genetic diseases that are characterized by progressive structural or functional changes of the muscles. Nursing treatment is to keep the function of respiratory levels and stimulate the quality of life. In this context, the objective of this article was to verify the performance of nursing in respiratory complications of DMD. This work consisted of a bibliographical review, whose search strategy included query-based electronic databases SciELO, Science Direct, Bireme and PEDro. The absence of studies involving the practice of nursing in Duchenne Muscular Dystrophy, the most common and severe among the hereditary neuromuscular diseases known, has led to not taking action consistent with the public and private authorities of scientific and medical societies. There is a need to know the performance of nursing in respiratory complications of DMD, so that this knowledge can contribute to more accurate and effective possible actions by public and private stakeholders who are directly or indirectly involved in the provision of health services, as well as promote the critical knowledge and humanistic training that can help students and professionals have relevant and true information.

\section{KEYWORDS}

Duchenne Muscular Dystrophy. Nursing. Respiratory complications

\section{INTRODUÇ̃̃̃}

A distrofia muscular de Duchenne corresponde a um grupo de doenças de origem genética progressiva que se caracterizam por alterações estruturais ou funcionais dos músculos (OTSUKA et al., 2005). 0 gene anormal localiza-se no braço curto do cromossomo $X$, que em condições normais é responsável pela

\section{RESUMEN}

La distrofia muscular es un grupo de enfermedades genéticas que se caracterizan por cambios progresivos estructurales o funcionales. El tratamiento de enfermería se basa, sobre todo, en mantener los niveles de las funciones respiratorias y estimular la calidad de vida. En éste contexto, el objetivo de este artículo es el de comprobar el rendimiento de la enfermería en las complicaciones respiratorias de DMD. Este trabajo consistió en una revisión bibliográfica, cuya estrategia de búsqueda, fue la de consultar los datos electrónica Scielo, Science Direct, Bireme y PEDro. La ausencia de estudios que involucran la práctica de enfermería en Distrofia Muscular de Duchenne, la más común y más grave entre las enfermedades neuromusculares hereditarias conocidas, ha tomado la decisión de actitudes no coherentes por autoridades públicas y privadas, sociedades médicas y científicas. Es importante tener conocimiento del rendimiento de enfermería en las complicaciones respiratorias de DMD, para que este conocimiento puede contribuir a la más precisas y eficaces posibles actuaciones por parte de actores públicos y privados, que participan directa o indirectamente en la prestación de servicios de salud, así como la promoción del conocimiento crítico y la formación humanista que pueda ayudar a estudiantes y profesionales a disponer de información verdadera y relevante.

\section{PALABRAS CLAVE}

Distrofia Muscular de Duchenne. Enfermería. Complicaciones respiratórias

produção de uma proteína denominada distrofina, situada no sarcolema das fibras musculares (SANTOS et al., 2006).

Atualmente, a incidência da DMD esta estimada em torno de 1:3.500 nascidos vivos do sexo mascu- 
lino. No Brasil, ocorrem por ano, cerca de 700 novos casos. Nos estudos realizados por Dooley (2010), embora em algumas situações possa afetar o gênero feminino na frequência de 1:2.500, a mulher possui dois cromossomos $X$, se um deles estiver afetado pelo erro genético, o outro compensa com a alteração e a doença não se manifesta, tornando esta uma portadora assintomática (BORGES et al., 2007; MACHADO, 2007; BAUMGARTNER e RUIZ, 2008; FACHARDO, 2004).

A DMD é considerada a mais comum e também a mais grave das distrofias musculares, sendo caracterizadas clinicamente por fraqueza e atrofia musculares progressivas, com grande variação quanto à intensidade do comprometimento e época do início (BORGES et al., 2007; NAIR et al., 2001; REED, 2002). Embora existam variações na evolução do quadro clínico, normalmente os portadores de DMD não são capazes de continuar deambulando após os 16 anos de idade (FREZZA et al., 2005; REED, 2002; OVANDO, 2008; GYPEL, 1996).

Do ponto de vista respiratório, a DMD frequentemente segue um curso previsível. 0 declínio progressivo da função pulmonar quase sempre inicia após o confinamento à cadeira de rodas e está associado com o aumento da insuficiência respiratória (IR) e da ineficiência da tosse, tornando os pacientes vulneráveis à atelectasias, pneumonias e retenção de secreções endobronquiais (OTSUKA et al., 2005). A insuficiência respiratória vem seguida de dificuldade na ventilação, falta de força para tossir, ocasionando infecções respiratórias de repetição, que na maioria dos casos leva o paciente a óbito (PETERLIN et al., 1997).

Pacientes com doença neuromuscular também apresentam anormalidade no padrão respiratório. Normalmente, o tórax e o abdômen se expandem simultaneamente durante a inspiração (SANTOS et al., 2006). Já nos pacientes com fraqueza muscular respiratória, ocorre uma projeção interna do tórax e uma projeção externa do abdômen durante a inspiração. Este padrão respiratório chamado de 'paradoxal' causa uma respiração ineficiente (OTSUKA et al., 2005). Deste modo, os pacientes com DMD têm um aumento no trabalho respiratório e uma expansibilidade pulmonar diminuída, resultando numa fadiga muscular respiratória, hipoventilação progressiva e potencial falência respiratória (MACHADO, 2007).

O músculo cardíaco também é afetado em praticamente todos os pacientes que apresentam maior sobrevida. 0 óbito pode ocorrer por volta dos 18 aos 25 anos por comprometimento cardíaco ou insuficiência respiratória Para seu diagnóstico são necessários alguns testes específicos. Primeiramente, verificam-se os níveis de uma enzima muscular chamada creatinaquiinase, que tem níveis relativamente baixos no sangue em condições normais. Pode ser realizado também biópsia muscular, onde um pequeno pedaço do músculo é removido (CAROMANO, 1998; MACHADO, 2007; OTSUKA, 2005).

Nesse contexto, o tratamento de Enfermagem é destinado a garantir a segurança física do cliente, promover o autocuidado, manter a nutrição adequada, assistência aos cuidadores e familiares. Nesse sentido, o enfermeiro exerce um papel fundamental, empenhando-se em preencher uma importante lacuna no estudo desta especialidade. (SANTOS et al., 2011).

É fundamental manter a função dos níveis respiratórios e estimular a qualidade de vida. As exigências físicas do paciente são consideráveis, sem perder de vista as necessidades emocionais e de desenvolvimento. O paciente e a família são ativamente envolvidos na tomada de decisão, incluindo as decisões de final de vida. As prescrições são destinadas a garantir a manutenção da segurança física do paciente, reduzir a ansiedade e agitação, estimular a independência das atividades de 
autocuidado, a manutenção da nutrição adequada, controle dos distúrbios e educação destinadas aos cuidadores e familiares. (SANTOS et al., 2011).

0 presente artigo teve como objetivos verificar a atuação da Enfermagem nas complicações respira- tórias da DMD, analisar os cuidados de Enfermagem, a manutenção da segurança física do paciente, bem como verificar assistência aos cuidadores e familiares quanto às complicações abordadas.

\section{METODOLOGIA}

Este trabalho consistiu em uma revisão bibliográfica, cuja estratégia de busca incluiu consulta à base de dados eletrônicas Scielo, Science Direct, e Pedro (Physiotherapy Evidence Database).

0 alvo dessa busca foram estudos com abordagem da Enfermagem na DMD. Para seleção, buscou-se a combinação dos termos distrofia muscular, enfermagem e complicações respiratórias. A busca foi realizada no período compreendido entre fevereiro e maio de 2013.

Para a inclusão dos artigos no estudo, foram definidos critérios a fim de atender adequadamente aos objetivos estabelecidos.

\section{DESENVOLVIMENTO}

As intervenções de Enfermagem delineiam um conjunto de atividades específicas ao serem executados os tratamentos de Enfermagem, e são tracejadas em resposta a um diagnóstico de Enfermagem pré-estabelecido (MONTEIRO et al., 2007). Segundo Lucena et al. (2010), o processo de Enfermagem é instrumento para o planejamento, a organização e a execução do cuidado de Enfermagem, onde os cuidados paliativos aumentam a qualidade de vida de pacientes e seus familiares que enfrentam uma doença terminal, através da prevenção e alívio do sofrimento (SANTANA et al., 2009).
Critérios para inclusão dos artigos: a) abordagem sobre DMD e doenças neuromusculares; b) discussão sobre formas de tratamento quanto às complicações respiratórias desse paciente da DM. Foram excluídos da presente pesquisa os artigos que abordaram apenas tratamentos medicamentosos ou cirúrgicos e publicados fora do período citado.

Os textos foram analisados e sintetizados de forma crítica, a fim de discutir as informações obtidas que correspondiam especificamente ao tema pretendido para compor esta revisão.

Para Silva (2008), o cuidado traz consigo a proposta de humanização do processo de desenvolver ações e serviços de saúde. Para tanto, a humanização não é mais uma prática adotada durante o atendimento ao paciente e sim uma visão generalista com troca de conhecimentos, experiências e sentimentos, apresentando-se como um ato de respeito e solidariedade (SANTANA et al., 2009).

Os cuidados humanizados com os pacientes de DMD na unidade de terapia intensiva resgatam a dignidade do ser humano, muitas vezes, abalada pela si- 
tuação em que se encontram. Entende-se, portanto, que ações simples como o toque, a conversa, a informação técnica referente ao setor, chamar o paciente pelo nome, tom de voz calmo com volume normal e a empatia dos profissionais podem contribuir significativamente para amenizar os efeitos nocivos da internação em UTI, evidenciando, assim, os aspectos positivos para os clientes e seus familiares, facilitando, desse modo, o processo de internação (SILVA e CONTRIN, 2007).

Diante da impossibilidade do autocuidado, as crianças realizam suas atividades com o auxílio das mães, estas cuidam de seus filhos da melhor forma, proporcionando uma qualidade de vida mais condizente e facilitando a adaptação da relação familiar com o cuidado à criança com DMD (OLIVEIRA et al., 2013). Nesse momento a Enfermagem objetiva ensinar o paciente no tratamento do autocuidado, onde o paciente e a família necessitam de informações e instruções sobre o distúrbio, sua evolução prevista e as estratégias de cuidado e tratamento que otimizarão o crescimento, desenvolvimento e estado físico e psicológico do paciente (SANTOS et al., 2013).

Muitas vezes, o paciente com DMD torna-se crítico e perde o contato direto com seus familiares, é destituído temporariamente da sociedade, de suas atividades e rotinas, tendo que se relacionar com desconhecidos e ficar exposto a situações constrangedoras, e pode enfrentar ainda fatores que geram medo e angústia e o deixam mais fragilizado e debilitado no seu estado emocional. Desse modo, o cuidado de Enfermagem com a família deve ir além de permitir a visita do familiar, deve incluir o estabelecimento de uma relação de confiança e de ajuda, na qual a equipe de Enfermagem tem a função de identificar as reais necessidades dos familiares, facilitando o tratamento do paciente (MEZZAROBA et al., 2009).

Nas doenças neuromusculares, principalmente na DMD, pode ocorrer insuficiência ventilatória que, quando associada às infecções pulmonares, constitui a causa mais frequente de morbidade e mortalidade (OTSUKA, 2005).

Em relação a esses comprometimentos respiratórios, técnicas como controle respiratório e relaxamento progressivo são essenciais para proporcionar redução da ansiedade, uma vez que os pacientes em fase final da doença apresentam dificuldades com relação a isso (SANTOS et al., 2013). Além dos cuidados com o paciente, há extrema preocupação quando aos seus cuidadores, que convivem diretamente com as complicações. Dessa forma, são ensinadas técnicas de autoinstrução para que possam organizar seus pensamentos e sentimentos quanto às possibilidades de evolução da doença de seus filhos, tornando-as conscientes das limitações de suas ações como cuidadores (COSTA; COSTA, 2007).

Além disso, são realizadas avaliações frequentes dos pacientes durante a vigília e o sono, associadas a sua respiração, com objetivo de promover a melhora, a força e a resistência da musculatura respiratória e diminuir anormalidades nas trocas gasosas (SANTOS et al., 2013), uma vez que com a evolução da doença, o tratamento das manifestações respiratórias requer melhora da ventilação, onde terapias para as complicações respiratórias de doenças neuromusculares pediátricas incluem métodos manuais e mecânicos de assistência à tosse assistida, respiração profunda e ventilação mecânica assistida (FONSECA; FRANCA, 2004).

Para Otsuka (2005), a reeducação da função muscular respiratória tem como objetivo principal ensinar e conscientizar o paciente a utilizar um padrão respiratório mais adequado, principalmente o diafragmático, no qual o paciente realizará a respiração de maneira mais econômica energeticamente e mais vantajosa mecânica e funcionalmente.

Para se reduzir as complicações respiratórias, o ideal é realizar acompanhamento médico periódico; ingerir líquidos, principalmente nos episódios de in- 
fecção respiratória, para tornar as secreções mais fluidas e facilitar a sua eliminação; controlar o excesso de peso ou perda de peso, que possa interferir no trabalho respiratório; manutenção da postura adequada na cadeira de rodas e nas atividades do dia a dia, fazendo adaptações na cadeira de rodas, se necessário (OTSUKA, 2005).

Como o tratamento de um paciente com uma doença neuromuscular grave é agressivo e apresenta pequena perspectiva de melhora clínica significativa, é um dilema ético indicar um programa terapêutico a estes sofridos pacientes. A indicação de um suporte respiratório e a escolha do método de ventilação numa complicação respiratória, por exemplo, pode necessitar de uma equipe multidisciplinar, incluindo a família e o próprio paciente. Como todo o tratamento de um paciente portador de distrofia muscular visa melhorar sua qualidade de vida, ultimamente, é o próprio paciente e seus familiares que determinam o que constitui uma aceitável qualidade de vida para eles, e assim, podem optar ou não pelo tratamento proposto (WEIRICH et al., 2004).

A doença neuromuscular e as deformidades associadas podem progredir na adolescência e na vida adulta. Os aparelhos de assistência e autoajuda auxiliam na manutenção da independência máxima. Dispositivos de autoajuda adicionais recomendados tornam-se, com frequência, necessários à medida que mais grupos musculares são afetados. 0 profissional de saúde pode auxiliar o paciente a adaptar-se com a doença, e compreender e abordar as necessidades físicas e psicológicas do paciente e família. A Enfermagem propicia um ambiente espaçoso de apoio e estímulos (SANTOS et al., 2013).

Estudos realizados por Pedrolo e Lima (2012) mostram que a qualidade de vida avaliada nos portadores com DMD revelaram que estes possuem menor pontuação em todos os domínios descritos, provavelmente pela patologia apresentar uma progressão mais rápi- da e acometer mais crianças. Já os estudos desenvolvidos por Gonçalves et al. (2008), utilizando questionário Auquei, indicaram uma boa qualidade de vida das crianças com DMD, tanto do ponto de vista das próprias crianças quanto de seus cuidadores.

Para a Organização Mundial da Saúde (OMS), a qualidade de vida consiste em bem-estar físico, mental e social e não somente na ausência de doenças ou enfermidade. A qualidade de vida dos pacientes portadores de distrofias ainda é considerada subjetiva, necessitando de mais pesquisas a respeito do assunto. Pode-se afirmar que a posição social e o conhecimento da patologia por esses indivíduos estão diretamente ligados à qualidade de vida que eles apresentam (PEDROLO; LIMA, 2012).

Para Gonçalves et al. (2008), embora a qualidade de vida desses pacientes seja muito discutida, a maioria dos estudos realizados sobre qualidade de vida em crianças aborda os familiares ou somente adolescentes, no entanto, esses dados não podem ser extrapolados para crianças. É importante avaliar a qualidade de vida através da ótica do próprio paciente e não apenas através dos adultos.

As complicações respiratórias diante do avanço tecnológico vêm permitindo prolongar suas vidas, mas nem sempre têm produzido impacto qualitativo. Nas doenças pulmonares, múltiplos fatores estão inter-relacionados e podem influenciar a percepção que as pessoas têm sobre sua saúde e a qualidade de vida. Há necessidade de desenvolvimento de educação em saúde em grupos como alternativa que tem trazido resultados efetivos na promoção da saúde, do bem-estar e do viver mais plenamente suas potencialidades (SILVA et al., 2005).

A prática de Enfermagem se destaca como a identificação de um conjunto de diagnósticos e intervenções de Enfermagem utilizada pelos enfermeiros em sua prática cotidiana, o que pode auxiliar na constru- 
ção de um corpo de conhecimentos, baseado em evidências sobre o cuidar de pacientes críticos, na elaboração de protocolos, na fundamentação do ensino e raciocínio clínico, no gerenciamento de custos e no planejamento de alocação de recursos para a qualificação dos serviços de Enfermagem (LUCENA et al., 2010).

\section{CONSIDERAÇÕES FINAIS}

A ausência de estudos envolvendo a prática de Enfermagem na Distrofia Muscular de Duchenne, a mais comum e mais grave dentre as doenças neuromusculares hereditárias conhecidas, tem levado a não tomada de atitudes coerentes por parte dos poderes públicos e privados, das sociedades médicas e científicas.
Dessa forma, ao inserir o cuidado de Enfermagem no âmbito político e ético, entendemos parte significativa na formação do enfermeiro com o estudo de uma visão holística. A política e o humanismo oferecem suportes para que a Enfermagem reafirme os valores, sentido e existência do cuidado que se preconiza como próprio desta prática profissional e que se legitima na convivência cidadã (SOUZA et al., 2005).

Há a necessidade de se conhecer a atuação da Enfermagem nas complicações respiratórias da DMD, para que este conhecimento possa contribuir para possíveis ações mais precisas e eficazes por parte de agentes públicos e privados que estão direta ou indiretamente envolvidos na prestação de serviços de saúde, bem como promover a formação de conhecimento crítico e humanístico que possa favorecer que estudantes e profissionais tenham informações verdadeiras e relevantes.

\section{REFERÊNCIAS}

BAUMGARTNER, M., RUIZ, D.A. Distrofia Muscular de Duchenne (Revision Bibliográfica). Revista médica de Costa Rica. v.65, n 586, 2008.

BORGES, D.; MOURA, E.W.; LIMA, E.; SILVA, P.A.C.. FISIOTERAPIA: Aspectos clínicos e práticos da reabilitação. 1. ed. São Paulo: Artes Medicas, 2007.

CAROMANO, F. A; et. al; Efeitos fisiológicos de sessão de hidroterapia em crianças portadoras de Distrofia Muscular de Duchenne. Revista Fisioterapia Universidade de São Paulo; São Paulo, v.5, n.1, p.49-55, jan-jun, 1998.

DOOLEY, J. GORDAN, K.E.; DODDS, L.; SWEEN, J.M. Duchenne muscular dystrophy a 30-year population based incidence study. Clinic Pediatric. v. 49, n. 2. March, 2010.

FACHARDO, G. A. Tratamento fisioterápico na distrofia muscular de Duchenne: Relato de Caso. Revista Neurociências, v.12, n. 4, 2004. 
FREZZA, R. M.; SILVA, S. R.,; FAGUNDES, S. L. Atualização do tratamento fisioterapêutico das distrofias musculares de Duchenne e Becker. RBPS, v. 18, n.1, 2005.

GYPEL, S.; DAMENT, A. Neurologia Infantil. 3. ed. Belo Horizonte: Atheneu, 1996. IWABE, C., MIRANDA, B.H., NUCCI, A. Medida da função motora: versão da escala para o português e estudo de confiabilidade. Revista Brasileira de Fisioterapia, v.12, n.5, 2008.

MACHADO, M. J. F. Distrofia muscular de Duchenne: complicações respiratórias e seu tratamento. Revista Ciências Médicas, v. 16, n. 2, 2007.

NAIR S. K. P., VASANTH, A.; GOURIE, D.M.; TALY, A.B.; RAO, S.; GAYATHRI, N.; MURALI, T. Disabilities in children with Duchenne muscular dystrophy: a profile. Journal Rehabilitation Medicine, v.33, 2001.

OMS, Organização Mundial de Saúde. Disponível em: < http://www.who.int/en/> Acesso em: 22 de mar. de 2013;

OTSUKA, M. A.; BOFFA, C.F.B.; VIEIRA, A.B.A.D.M. Distrofias Musculares: Fisioterapia Aplicada. 2. ed. São Paulo: Revinter, 2005.

OVANDO, A. C. A Hidroterapia como forma de tratamento para a distrofia muscular de Duchenne: Relato de caso. Revista Digital, v 13, n. 126, 2008.

PETERLIN, B.; ZIDAR, J.; MEZNARIC, P.M.; ZUPANCIC, N. Genetic epidemiology in Duchenne and Becker muscular dystrophy in Slovenia. Clinic Genetic, march, v. 51, n. 2, 1997.

REED, U. C. Doenças neuromusculares. Jornal de Pediatria. v. 78, n.1., 2009.

SANTOS, F.P.O.; AGUIAR, V.R.F.; MANCEBO, W.G.N.; CONTENÇAS, T.S. Assistência de enfermagem nas complicações respiratórias da distrofia muscular de Duchenne. Disponível em: <http://www.webartigos.com/ artigos/assistencia-de-enfermagem-nas-complicacoes-respiratoriasda-distrofia-muscular-de-duchenne/67925/> Acesso em: mar. de 2013.

SANTOS, N. M.; REZENDE, M.M.; TERNI, A.; HAYASHI, M.C.B.; FAVERO, F.M.; QUADROS, A.A.J.; REIS, L.I.O.; ADISSI, M.; LANGER, A.L.; FONTES, S.V.; OLIVEIRA, A.S.B. Perfil clínico e funcional dos pacientes com Distrofia Muscular de Duchenne assistidos na associação brasileira de distrofia muscular (ABDIM). Revista Neurociências, v. 14, n.1, 2006.

SILVA, M.G.G. Doença terminal, perspectiva de morte: um trabalho desafiador ao profissional da saúde que luta contra ela. Rev SBPH v. 2, n. , 2005;

SOUZA, M. L. ; SARTOR, V.V.B.; PADILHA, M.I.C.S.; PRADO, M.L. O cuidado em Enfermagem - Uma aproximação teórica. Texto Contex Enferm, v. 14, n. 2, 2005. 
